\title{
(1+1)-Dimensional Yang-Mills Theory Coupled to Adjoint Fermions on the Light Front
}

\author{
Stephen S. Pinsky \\ Department of Physics, The Ohio State University, Columbus, OH 43210
}

\begin{abstract}
We consider $S U(2)$ Yang-Mills theory in $1+1$ dimensions coupled to massless adjoint fermions. With all fields in the adjoint representation the gauge group is actually $S U(2) / Z_{2}$, which possesses nontrivial topology. In particular, there are two distinct topological sectors and the physical vacuum state has a structure analogous to a $\theta$ vacuum. We show how this feature is realized in light-front quantization, with periodicity conditions in $x^{ \pm}$used to regulate the infrared and treating the gauge field zero mode as a dynamical quantity. We find expressions for the degenerate vacuum states and construct the analog of the $\theta$ vacuum. We then calculate the bilinear condensate in the model. We argue that the condensate does not affect the spectrum of the theory, although it is related to the string tension that characterizes the potential between fundamental test charges when the dynamical fermions are given a mass. We also argue that this result is fundamentally different from calculations that use periodicity conditions in $x^{1}$ as an infrared regulator.
\end{abstract}




\section{INTRODUCTION}

The unique features of light-front quantization [四] make it a potentially powerful tool for the nonperturbative study of quantum field theories. The main advantage of this approach is the apparent simplicity of the vacuum state. Indeed, naive kinematical arguments suggest that the physical vacuum is trivial on the light front. It is difficult to imagine that this can really be true, particularly in light of the important physics associated with the vacuum in QCD. Thus it is crucial to understand the ways in which vacuum structure can be manifested in light-front quantization.

This problem has recently been discussed in a variety of contexts. If one uses discretization as an infrared regulator (i.e., imposes periodic or antiperiodic boundary conditions on some finite interval in $x^{-}$, the so called discrete light-cone quantization (DLCQ) approach [2]) then any dynamical vacuum structure must necessarily be connected with the $k^{+}=0$ Fourier modes of the fields. Studies of model field theories have shown that certain aspects of vacuum physics can in fact be reproduced by a careful treatment of the field zero modes. For example, solutions of the zero mode constraint equation in $\phi_{1+1}^{4}$ [3] exhibit spontaneous symmetry breaking [4].

We shall focus our attention here on $S U(2)$ Yang-Mills theory coupled to adjoint fermions. This theory is known to have nontrivial vacuum structure; as first pointed out in [9], for $S U(N)$ gauge fields the model has a $Z_{N}$ topological structure and a physical vacuum state analogous to a $\theta$ vacuum. In this theory the vacuum structure arises because of symmetry considerations and it is therefore fundamentally different from the $\phi_{1+1}^{4}$ light-front theories. In addition, for $N=2$ there is a nonvanishing bilinear condensate [10]. This theory is interesting to study for a variety of additional reasons. While it differs significantly from the case of $\mathrm{QCD}_{1+1}$ with fundamental matter, where the topology is trivial and there is a unique vacuum state, it has recently been shown that the massive spectra of these theories are related [11]. Adjoint fermions are also an important part of the first $(3+1)$-dimensional theories where confinement has been proven [12].

Our goal is to show how this structure is realized in light-front quantization, using periodicity conditions to regulate the theory. In this framework it is necessary to introduce degrees of freedom initialized along two different null planes [13] and to carefully define singular operator products in a gauge-invariant manner. Finally, one must pay proper attention to the effects of the boundary conditions on the choice of gauge. The most important issues here concern the proper treatment of the gauge field zero modes. In particular, for $S U(2)$ a zero mode of $A^{+}$must be retained in the theory. This quantity can be treated as a dynamical variable, and this is the approach we shall take here. A complementary formalism, in which this operator is treated as constrained, is discussed elsewhere [14]. We shall find two degenerate vacua and a nonvanishing condensate in the physical ground state. Furthermore, the vacuum states can be described completely, unlike in the equal-time representation. In this sense the vacuum structure is simpler on the light front than at equal time.

We will argue that the condensate we find is not related to that computed at equal time in [15]. We also briefly discuss the implications of our work for the universality of twodimensional gauge theories quantized on a light-cone spatial circle [11. We will show that the condensate does not affect the massive spectrum of the theory with massless fermions. It is however related to the string tension in the theory with massive adjoint fermions [16]. 


\section{II. $S U(2)$ GAUGE THEORY COUPLED TO ADJOINT FERMIONS}

We consider $S U(2)$ gauge theory coupled to adjoint fermions in one space and one time dimension. Since all fields transform according to the adjoint representation, gauge transformations that differ by an element of the center of the group actually represent the same transformation and so should be identified. Thus the gauge group of the theory is $S U(2) / Z_{2}$, which has nontrivial topology: $\Pi_{1}\left[S U(2) / Z_{2}\right]=Z_{2}$, so that we expect two topological sectors. This situation differs from the case when the matter fields are in the fundamental representation, where the gauge group is $S U(2)$ and the first homotopy group is trivial [17].

The Lagrangian for the theory is

$$
\mathcal{L}=-\frac{1}{2} \operatorname{Tr}\left(F^{\mu \nu} F_{\mu \nu}\right)+\frac{i}{2} \operatorname{Tr}\left(\bar{\psi} \gamma^{\mu} \stackrel{\leftrightarrow}{D}_{\mu} \psi\right)
$$

where $D_{\mu}=\partial_{\mu}+i g\left[A_{\mu}, \quad\right]$ and $F_{\mu \nu}=\partial_{\mu} A_{\nu}-\partial_{\nu} A_{\mu}+i g\left[A_{\mu}, A_{\nu}\right]$. A convenient representation of the gamma matrices is $\gamma^{0}=\sigma^{2}$ and $\gamma^{1}=i \sigma^{1}$, where $\sigma^{a}$ are the Pauli matrices. With this choice the Fermi field may be taken to be hermitian.

The matrix representation of the fields makes use of the $S U(2)$ generators $\tau^{a}=\sigma^{a} / 2$. It is convenient to introduce a color helicity, or Cartan, basis, defined by

$$
\tau^{ \pm} \equiv \frac{1}{\sqrt{2}}\left(\tau^{1} \pm i \tau^{2}\right)
$$

with $\tau^{3}$ unchanged. Lower helicity indices are defined by $\tau_{ \pm}=\tau^{\mp}$. These satisfy

$$
\begin{aligned}
& {\left[\tau^{+}, \tau^{-}\right]=\tau^{3}} \\
& {\left[\tau^{3}, \tau^{ \pm}\right]= \pm \tau^{ \pm} .}
\end{aligned}
$$

In terms of this basis, matrix-valued fields are decomposed as, for example,

$$
A^{\mu}=A_{3}^{\mu} \tau^{3}+A_{+}^{\mu} \tau^{+}+A_{-}^{\mu} \tau^{-},
$$

where $A^{\mu, \pm} \equiv\left(A_{1}^{\mu} \pm i A_{2}^{\mu}\right) / \sqrt{2}$ and $A^{\mu, \pm}=A_{\mp}^{\mu}$. (Note also that $\left(A_{+}^{\mu}\right)^{\dagger}=A_{-}^{\mu}$.) The Fermi field will be written as

$$
\Psi_{R / L}=\psi_{R / L} \tau^{3}+\phi_{R / L} \tau^{+}+\phi_{R / L}^{\dagger} \tau^{-}
$$

where $\phi_{R / L} \equiv\left(\Psi_{R / L}^{1}-i \Psi_{R / L}^{2}\right) / \sqrt{2}$ and the labels $R / L$ indicate light-front spinor projections. Note that under a gauge transformation the Fermi field transforms according to

$$
\Psi_{R / L}^{\prime}=U \Psi_{R / L} U^{-1}
$$

where $U$ is a spacetime-dependent element of $S U(2)$. The gauge field transforms in the conventional way.

We shall regulate the theory by requiring that the gauge field $A^{\mu}$ be periodic and the right-handed Fermi field be antiperiodic in $x^{-}$. The left-handed Fermion $\Psi_{L}$ is taken to be antiperiodic in the coordinate $x^{+}$. In this "discretized" formulation, most of the subtleties have to do with the zero-momentum Fourier modes of the fields. 
The Fock space representation for the Fermi fields is obtained by Fourier expanding $\Psi_{R}$ on $x^{+}=0$ and $\Psi_{L}$ on $x^{-}=0$ :

$$
\begin{aligned}
\psi_{R} & =\frac{1}{2^{1 / 4} \sqrt{2 L}} \sum_{n}\left(a_{n} e^{-i k_{n}^{+} x^{-}}+a_{n}^{\dagger} e^{i k_{n}^{+} x^{-}}\right) \\
\phi_{R} & =\frac{1}{2^{1 / 4} \sqrt{2 L}} \sum_{n}\left(b_{n} e^{-i k_{n}^{+} x^{-}}+d_{n}^{\dagger} e^{i k_{n}^{+} x^{-}}\right) \\
\psi_{L} & =\frac{1}{2^{1 / 4} \sqrt{2 L}} \sum_{n}\left(\alpha_{n} e^{-i k_{n}^{-} x^{+}}+\alpha_{n}^{\dagger} e^{i k_{n}^{-} x^{+}}\right) \\
\phi_{L} & =\frac{1}{2^{1 / 4} \sqrt{2 L}} \sum_{n}\left(\beta_{n} e^{-i k_{n}^{-} x^{+}}+\delta_{n}^{\dagger} e^{i k_{n}^{-} x^{+}}\right) .
\end{aligned}
$$

Here the sums run over the positive half-odd integers and $k_{n}^{ \pm}=n \pi / L$. The Fourier modes obey the standard anti-commutation relations

$$
\begin{gathered}
\left\{a_{n}^{\dagger}, a_{m}\right\}=\left\{b_{n}^{\dagger}, b_{m}\right\}=\left\{d_{n}^{\dagger}, d_{m}\right\}=\delta_{n, m} \\
\left\{\alpha_{n}^{\dagger}, \alpha_{m}\right\}=\left\{\beta_{n}^{\dagger}, \beta_{m}\right\}=\left\{\delta_{n}^{\dagger}, \delta_{m}\right\}=\delta_{n, m},
\end{gathered}
$$

with all mixed anti-commutators vanishing. These are equivalent to the canonical anticommutators

$$
\begin{aligned}
& \left\{\Psi_{R}\left(0, x^{-}\right), \Psi_{R}\left(0, y^{-}\right)\right\}=\frac{1}{\sqrt{2}} \delta\left(x^{-}-y^{-}\right) \\
& \left\{\Psi_{L}\left(x^{+}, 0\right), \Psi_{L}\left(y^{+}, 0\right)\right\}=\frac{1}{\sqrt{2}} \delta\left(x^{+}-y^{+}\right) .
\end{aligned}
$$

The fermionic Fock space is generated by acting with the various creation operators on a vacuum state $|0\rangle$.

\section{CURRENT OPERATORS}

The current operators for this theory are

$$
\begin{aligned}
J^{+} & \equiv J^{R}=-\frac{1}{\sqrt{2}}\left[\Psi_{R}, \Psi_{R}\right] \\
J^{-} & \equiv J^{L}=-\frac{1}{\sqrt{2}}\left[\Psi_{L}, \Psi_{L}\right] .
\end{aligned}
$$

To avoid confusion, we shall henceforth always write the currents with $R$ or $L$ in place of the upper Lorentz index and the color helicity index either up or down with $J_{3}=J^{3}$ and $J_{-}=J^{+}$.

These expressions for the currents are ill-defined as they stand since they contain the product of operators at the same point. This is a common problem and occurs in the expression for the Poincaré generators as well. We shall regulate these expressions by point 
splitting while maintaining gauge invariance and then take the splitting to zero after removing the singularities. The singularities give rise to additional contributions, so called gauge corrections. To maintain gauge invariance in the split product one must introduce an eikonal factor. One can show for example that

$$
\left[e^{i g \int_{x}^{x+\epsilon^{\mu}} A \cdot d x} \Psi\left(x+\epsilon^{\mu}\right) e^{-i g \int_{x}^{x+\epsilon^{\mu}} A \cdot d x}, \Psi(x)\right]
$$

transforms canonically in the adjoint representation. In the limit of small $\epsilon^{\mu}$ one effectively replaces

$$
\psi(x) \rightarrow \psi\left(x+\epsilon^{\mu}\right)+i g\left[A \cdot \epsilon, \psi\left(x+\epsilon^{\mu}\right)\right]
$$

The singularity in the operator product as $\epsilon \rightarrow 0$ picks up the $\epsilon$ in the above expression leaving an additional contribution. The splitting must be performed in the $x^{-}$direction for $\Psi_{R}$. We find that the current $J^{R}$ acquires a gauge correction

$$
J^{R}=\tilde{J}^{R}-\frac{g}{2 \pi} V,
$$

where $\tilde{J}^{R}$ is the naive normal-ordered current. The operator product in $J^{L}$ must be split in the $x^{+}$direction, and an analogous calculation gives

$$
J^{L}=\tilde{J}^{L}-\frac{g}{2 \pi} A
$$

where $\tilde{J}^{L}$ is the naive normal-ordered current.

In the helicity basis, $\tilde{J}^{R}$ takes the form

$$
\begin{aligned}
& \tilde{J}_{3}^{R}=: \frac{1}{\sqrt{2}}\left(\phi_{R}^{\dagger} \phi_{R}-\phi_{R} \phi_{R}^{\dagger}\right): \\
& \tilde{J}_{-}^{R}=: \frac{1}{\sqrt{2}}\left(\psi_{R} \phi_{R}^{\dagger}-\phi_{R}^{\dagger} \psi_{R}\right): \\
& \tilde{J}_{+}^{R}=: \frac{1}{\sqrt{2}}\left(\phi_{R} \psi_{R}-\psi_{R} \phi_{R}\right): .
\end{aligned}
$$

The corresponding expressions for the components of $\tilde{J}^{L}$ are identical, with $R \rightarrow L$. It is convenient to Fourier expand these currents and discuss the the properties of their components. We write

$$
\begin{aligned}
\tilde{J}^{R, a} & =\frac{1}{2 L} \sum_{N=-\infty}^{\infty} C_{N}^{a} e^{-i \pi N x^{-} / L} \\
\tilde{J}^{L, a} & =\frac{1}{2 L} \sum_{N=-\infty}^{\infty} D_{N}^{a} e^{-i \pi N x^{+} / L},
\end{aligned}
$$

where $a$ is a color index and the sums are over the integers. It is well known that the Fourier components satisfy a Kac-Moody algebra with level one [18]. We shall discuss this explicitly for the $C_{N}^{a}$; an identical set of relations holds for the $D_{N}^{a}$, with appropriate substitutions. 
In terms of the Fock operators, we find for $N>0$

$$
\begin{aligned}
& C_{N}^{3}=\sum_{n=\frac{1}{2}}^{\infty} b_{n}^{\dagger} b_{N+n}-\sum_{n=\frac{1}{2}}^{\infty} d_{n}^{\dagger} d_{N+n}-\sum_{n=\frac{1}{2}}^{N-\frac{1}{2}} b_{n} d_{N-n} \\
& C_{N}^{+}=\sum_{n=\frac{1}{2}}^{\infty} a_{n}^{\dagger} d_{N+n}-\sum_{n=\frac{1}{2}}^{\infty} b_{n}^{\dagger} a_{N+n}-\sum_{n=\frac{1}{2}}^{N-\frac{1}{2}} d_{n} a_{N-n} \\
& C_{N}^{-}=\sum_{n=\frac{1}{2}}^{\infty} d_{n}^{\dagger} a_{N+n}-\sum_{n=\frac{1}{2}}^{\infty} a_{n}^{\dagger} b_{N+n}-\sum_{n=\frac{1}{2}}^{N-\frac{1}{2}} a_{n} b_{N-n} .
\end{aligned}
$$

The negative frequency modes may be obtained by hermitian conjugation:

$$
C_{-N}^{3}=\left(C_{N}^{3}\right)^{\dagger} \quad C_{-N}^{+}=\left(C_{N}^{-}\right)^{\dagger} \quad C_{-N}^{-}=\left(C_{N}^{+}\right)^{\dagger} .
$$

Finally, the $N=0$ terms are just the various color charges in the right-moving fermions:

$$
\begin{aligned}
C_{0}^{3} & =\sum_{n}\left(b_{n}^{\dagger} b_{n}-d_{n}^{\dagger} d_{n}\right) \equiv Q_{R} \\
C_{0}^{-} & =\sum_{n}\left(d_{n}^{\dagger} a_{n}-a_{n}^{\dagger} b_{n}\right) \\
C_{0}^{+} & =\sum_{n}\left(a_{n}^{\dagger} d_{n}-b_{n}^{\dagger} a_{n}\right) .
\end{aligned}
$$

In the Cartan basis, the Kac-Moody algebra takes the form

$$
\begin{aligned}
& {\left[C_{N}^{3}, C_{M}^{3}\right]=N \delta_{N,-M}} \\
& {\left[C_{N}^{ \pm}, C_{M}^{ \pm}\right]=0} \\
& {\left[C_{N}^{3}, C_{M}^{ \pm}\right]= \pm C_{N+M}^{ \pm}} \\
& {\left[C_{N}^{+}, C_{M}^{-}\right]=C_{N+M}^{3}+N \delta_{N,-M} .}
\end{aligned}
$$

It is straightforward to verify these relations using the fundamental anticommutators (2.12) and (2.13). The algebra satisfied by the $D_{N}^{a}$ is of course identical. Note that for $N=M=0$ the above algebra is the $S U(2)$ algebra of the charges.

\section{GAUGE FIXING}

In the present context, the main subtlety arising from discretization is in fixing the gauge. It is most convenient in light-front field theory to choose the light-cone gauge $A^{+} \equiv V=0$. This is not possible with the boundary conditions we have imposed, however; since the gauge transformation must be periodic up to an element of the center of the gauge group (here $Z_{2}$ ), we cannot gauge the zero mode of $V$ to zero 19. It is permissible to take $\partial_{-} V=0$. In addition, we can make a further global (i.e., $x^{-}$-independent) rotation so that the zero mode of $V$ has only a color 3 component, 


$$
V=v\left(x^{+}\right) \tau^{3}
$$

Note that the gauge transformation required to achieve this is $x^{+}$-dependent. In addition, the gauge field satisfies no particular boundary conditions in $x^{+}$. Thus the necessary transformation can be expected to conflict with the boundary condition imposed on $\Psi_{L}$. However, we can apply a purely $x^{+}$-dependent gauge transformation that restores the antiperiodicity of $\psi_{L}$ on its initial-value surface $x^{-}=0$.

While one could simultaneously rotate $A^{-} \equiv A$ so that it has no color 3 zero mode 20,21, we shall not do that here. Instead we will retain this zero mode, which we call $w\left(x^{+}\right)$, and determine it in the solution of the equations of motion.

At this stage the only remaining gauge freedom involves certain "large" gauge transformations which we shall denote $T_{N}^{R}$ and $T_{N}^{L}$, with $N$ any integer:

$$
\begin{aligned}
& T_{N}^{R}=\exp \left[-\frac{i N \pi}{2 L} x^{-} \tau_{3}\right] \\
& T_{N}^{L}=\exp \left[\frac{i N \pi}{2 L} x^{+} \tau_{3}\right] .
\end{aligned}
$$

This freedom is best studied in terms of the dimensionless variables $Z_{R}=g v L / \pi$ and $Z_{L}=g w L / \pi$, which $T_{N}^{R / L}$ shifts by $\pm N$ :

$$
\begin{aligned}
T_{N}^{R} Z_{R}\left(T_{N}^{R}\right)^{-1} & =Z_{R}+N \\
T_{N}^{L} Z_{L}\left(T_{N}^{L}\right)^{-1} & =Z_{L}-N .
\end{aligned}
$$

In addition, $T_{N}^{R / L}$ generates a space-time-dependent phase rotation on the matter field $\phi_{R / L}$,

$$
\begin{aligned}
T_{N}^{R} \phi_{R}\left(T_{N}^{R}\right)^{-1} & =e^{-i N \pi x^{-} / L} \phi_{R} \\
T_{N}^{L} \phi_{L}\left(T_{N}^{L}\right)^{-1} & =e^{i N \pi x^{+} / L} \phi_{L},
\end{aligned}
$$

which however preserves the boundary conditions on $\phi_{R / L}$. The combination $T_{N}^{R} T_{N}^{L} \equiv T_{N}$ is a gauge freedom and connects different Gribov copies in the theory [22]. We can use it to bring $Z_{R}$ to a "fundamental modular domain" (FMD), for example $-1<Z_{R}<0$. Once this is done all gauge freedom has been exhausted and the gauge fixing is completed.

The physical degrees of freedom that remain are the Fermi fields $\Psi_{R}$ and $\Psi_{L}$ and the gauge field zero mode $Z_{R}$, restricted to the finite interval $-1<Z_{R}<0$. All other nonvanishing components of the gauge field will be found to be constrained, as is usual in light-front field theory. Because of the finite domain of $Z_{R}$, it is convenient to use a Schrödinger representation for this variable. Thus the states of the theory will be written in the form

$$
\left.|\Phi\rangle=\zeta\left(Z_{R}\right) \mid \text { Fock }\right\rangle
$$

where $\zeta\left(Z_{R}\right)$ is a Schrödinger wavefunction on the FMD and $\mid$ Fock $\rangle$ is a Fock state in the fermionic variables. There remains the question of what boundary conditions should be satisfied by the wavefunction $\zeta$. A careful analysis of the integration measure used to define the inner product shows that there is a Jacobian factor which essentially forces the wavefunction to vanish at the boundaries of the fundamental domain [23]. Thus we have 


$$
\zeta(-1)=\zeta(0)=0
$$

After gauge fixing $T_{N}$ is no longer a symmetry of the theory, but there is an important symmetry of the gauge-fixed theory that is conveniently studied by combining $T_{1}$ with the so-called Weyl transformation, denoted by $R$. Under $R$,

$$
\begin{aligned}
& R Z_{R / L} R^{-1}=-Z_{R / L} \\
& R \phi_{R / L} R^{-1}=\phi_{R / L}^{\dagger} .
\end{aligned}
$$

$R$ is also not a symmetry of the gauge-fixed theory, as it takes $Z_{R}$ out of the fundamental domain. However, the combination $T_{1} R$ is a symmetry; from Eqs. (4.4) and (4.10) we have

$$
\begin{aligned}
& T_{1} R Z_{R} R^{-1} T_{1}^{-1}=-Z_{R}-1 \\
& T_{1} R Z_{L} R^{-1} T_{1}^{-1}=-Z_{L}+1,
\end{aligned}
$$

so that $T_{1} R$ maps the FMD $-1<Z_{R}<0$ onto itself. In fact, it represents a reflection of the FMD about its midpoint $Z_{R}=-1 / 2$.

The action of $T_{1}^{R / L}$ and $R$ on the fermion Fock operators can be determined from Eqs. (4.6), (4.7) and (4.11). $T_{1}^{R}$ gives rise to a spectral flow for the right handed fields,

$$
\begin{aligned}
T_{1}^{R} b_{n} T_{1}^{-1 R} & =b_{n-1} \quad(n>1 / 2) \\
T_{1}^{R} d_{n} T_{1}^{-1 R} & =d_{n+1} \\
T_{1}^{R} b_{1 / 2} T_{1}^{-1 R} & =d_{1 / 2}^{\dagger},
\end{aligned}
$$

while $T_{1}^{L}$ gives rise to spectral flow for the left handed fields,

$$
\begin{aligned}
T_{1}^{L} \beta_{n} T_{1}^{-1 L} & =\beta_{n+1} \\
T_{1}^{L} \delta_{n} T_{1}^{-1 L} & =\delta_{n-1} \quad(n>1 / 2) \\
T_{1}^{L} \delta_{1 / 2} T_{1}^{-1 L} & =\beta_{1 / 2}^{\dagger} .
\end{aligned}
$$

$R$ is analogous to charge conjugation:

$$
\begin{aligned}
& R b_{n} R^{-1}=-d_{n} \\
& R \beta_{n} R^{-1}=-\delta_{n}
\end{aligned}
$$

The $a_{n}$ and $\alpha_{n}$ are invariant under both $T_{1}^{R / L}$ and $R$. From the behavior of the Fock operator it is straightforward to deduce the behavior of the elements of the Kac Moody algebra under $T^{R}, T^{L}$ and $R$ and show that the algebra is invariant. We shall elaborate on the detailed implications of the symmetry $T_{1} R$ when we consider the structure of the vacuum state below.

Finally, let us discuss Gauss' law and determine the rest of the vector potential in terms of the dynamical degrees of freedom. Gauss's law in matrix form is

$$
D_{-}^{2} A=-g J^{R}
$$

In the Cartan basis this becomes 


$$
\begin{array}{r}
-\partial_{-}^{2} A_{3}=g J_{3}^{R} \\
-\left(\partial_{-} \pm i g v\right)^{2} A_{ \pm}=g J_{ \pm}^{R} .
\end{array}
$$

This is the reason for introducing the color helicity basis: in the gauge we have chosen, Gauss' law separates and its individual components can be solved directly. Note that because of the gauge choice, $J^{R}$ only acquires a gauge correction to its 3 color component. All color components of $J^{L}$ receive a gauge correction.

Eqn. (4.19) can be used to obtain the normal mode part of $A_{3}$ on the surface $x^{+}=0$ :

$$
A_{3}=\frac{g L}{2 \pi^{2}} \sum_{N \neq 0} \frac{C_{N}^{3}}{N^{2}} e^{-i N \pi x^{-} / L} .
$$

The zero mode of $A_{3}$ is not determined from Eqn. (4.19). We shall return to this in a moment, but for now note that since there is no zero mode on the left hand side of Eqn. (4.19), the zero mode of the right hand side must also vanish. This gives the relation

$$
0=Q_{R}-Z_{R}\left(x^{+}\right),
$$

Clearly this can not hold as an operator relation. Eventually this condition must be satisfied by restriction to a physical subspace. We shall discuss this in detail below.

Because of the restriction of $Z_{R}$ to the domain $[-1,0]$ and the boundary condition on $\zeta\left(Z_{R}\right)$, the covariant derivatives appearing in Eqn. (4.20) have no zero eigenvalues. Thus they may be inverted to give

$$
A_{ \pm}=\frac{g L}{2 \pi^{2}} \sum_{N} \frac{C_{N}^{\mp}}{\left(N \mp Z_{R}\right)^{2}} e^{-i N \pi x^{-} / L} .
$$

\section{POINCARÉ GENERATORS}

The Poincaré generators $P^{-}$and $P^{+}$have contribution from both the left handed and right handed fermions but $\Psi_{R}$ is initialized on $x^{+}=0$ and propagates in $x^{+}$while $\Psi_{L}$ is initialized on $x^{-}=0$ and propagates in $x^{-}$. These operators must therefore have contributions from both parts of the initial-value surface. Thus

$$
P^{ \pm}=\int_{-L}^{L} d x^{-} \Theta^{+ \pm}+\int_{-L}^{L} d x^{+} \Theta^{- \pm},
$$

where $\Theta^{\mu \nu}$ is the energy momentum tensor. There are two common forms for this, the Noether form and the symmetric gauge-invariant form. It is instructive to consider both. The standard Noether procedure gives

$$
\begin{aligned}
& \Theta^{+-}=-2 \operatorname{Tr}\left(F^{+-} \partial_{+} A^{+}\right)-\operatorname{Tr}\left(F^{+-} F^{+-}\right)+\frac{i}{\sqrt{2}} \operatorname{Tr}\left(\Psi_{R} \partial^{-} \Psi_{R}-\partial^{-} \Psi_{R} \Psi_{R}\right) \\
& \Theta^{-+}=-2 \operatorname{Tr}\left(F^{+-} \partial_{-} A^{-}\right)-\operatorname{Tr}\left(F^{+-} F^{+-}\right)+\frac{i}{\sqrt{2}} \operatorname{Tr}\left(\Psi_{L} \partial^{+} \Psi_{L}-\partial^{+} \Psi_{L} \Psi_{L}\right)
\end{aligned}
$$




$$
\begin{aligned}
& \Theta^{++}=\frac{i}{\sqrt{2}} \operatorname{Tr}\left(\Psi_{R} \partial^{+} \Psi_{R}-\partial^{+} \Psi_{R} \Psi_{R}\right) \\
& \Theta^{--}=\frac{i}{\sqrt{2}} \operatorname{Tr}\left(\Psi_{L} \partial^{-} \Psi_{L}-\partial^{-} \Psi_{L} \Psi_{L}\right)-2 \operatorname{Tr}\left(F^{-+} \partial_{+} A^{-}\right) .
\end{aligned}
$$

We see that both $\Theta^{-+}$and $\Theta^{--}$contain fields that are initialized on both $x^{+}=0$ and $x^{-}=0$. What are we to do with the parts that are initialized on $x^{-}=0$ ? One guess is simply to throw them away. Now consider the symmetric gauge-invariant form,

$$
\begin{aligned}
& \Theta^{+-}=\operatorname{Tr}\left(F^{+-} F^{+-}\right) \\
& \Theta^{-+}=\operatorname{Tr}\left(F^{+-} F^{+-}\right) \\
& \Theta^{++}=\frac{i}{\sqrt{2}} \operatorname{Tr}\left(\Psi_{R} D^{+} \Psi_{R}-D^{+} \Psi_{R} \Psi_{R}\right) \\
& \Theta^{--}=\frac{i}{\sqrt{2}} \operatorname{Tr}\left(\Psi_{L} D^{-} \Psi_{L}-D^{-} \Psi_{L} \Psi_{L}\right) .
\end{aligned}
$$

Again $\Theta^{--}$and $\Theta^{+-}$are ambiguous and if we use our rule of simply dropping terms then there is no reason to believe that these two forms are equivalent or that either is correct. Given this unpleasant situation we chose to use the symmetric gauge-invariant form and and apply the rule of dropping terms that are not initialized on the surface that they are to be integrated on. In the end we will justify this decision by checking that these generators result in the correct equation of motion.

First let us construct $P^{-}$. The contribution from the $x^{+}=0$ surface is the standard form one expects in theories of $Q C D_{1+1}$ coupled to matter and includes the contribution from the zero mode of $A^{+}$. In the contribution from the $x^{-}=0$ surface we will drop the terms containing $A$ in the covariant derivative. This leaves us with the same contribution that we would obtain from the Noether current applying the rule and this term give the momentum of the left movers which propagate in the $x^{-}$direction. We thus obtain

$$
P^{-}=-g^{2} \int_{-L}^{L} d x^{-} \operatorname{Tr}\left(J^{+} \frac{1}{D_{-}^{2}} J^{+}\right)+L\left(\partial_{+} v\right)^{2}++\frac{i}{\sqrt{2}} \int_{-L}^{L} d x^{+} \operatorname{Tr}\left(\Psi_{L} \partial_{+} \Psi_{L}+\partial_{+} \Psi_{L} \Psi_{L}\right) .
$$

Let us consider the left and right handed parts of this expression separately for a moment. The operator products appearing in the left-handed part are singular and require regulariztion and renormalization. We have done this in two ways, using the gauge-invariant point-splitting discussed earlier and also a $\zeta$-function regularization. Both procedures give the same result:

$$
P_{l h}^{-}=\frac{\pi}{L} \sum_{n}^{\prime} n\left(\alpha_{n}^{\dagger} \alpha_{n}+\beta_{n}^{\dagger} \beta_{n}+\delta_{n}^{\dagger} \delta_{n}\right)-\frac{\pi}{L} Z_{L} Q_{L}+\frac{\pi}{2 L} Z_{L}^{2}
$$

We will have more to say about these extra term as we further develop this theory.

Now consider the right handed contribution. This takes its most elegant form by expressing the $J^{R} \mathrm{~S}$ in term of the $C^{N} \mathrm{~s}$. We find 


$$
P_{r h}^{-}=\frac{g^{2} L}{4 \pi^{2}}\left[\sum_{N \neq 0} \frac{C_{N}^{3} C_{-N}^{3}}{N^{2}}+\sum_{N}\left[\frac{C_{N}^{+} C_{-N}^{-}}{\left(Z_{R}+N\right)^{2}}+\frac{C_{N}^{-} C_{-N}^{+}}{\left(Z_{R}-N\right)^{2}}\right]+\Pi_{R}^{2}\right]
$$

where $\Pi_{R}=(2 \pi / g) \partial_{+} v$ is the momentum conjugate to $Z_{R}$, so that $\left[Z_{R}, \Pi_{R}\right]=i$.

Turning now to the operator $P^{+}$we see that it only gets contributions from the $x^{+}=0$ surface when using the symmetric gauge invariant form. Thus the longitunial momentum operator is given by

$$
P^{+}=\frac{g}{\sqrt{2}} \int_{L}^{L} d x^{-} \operatorname{Tr}\left(\Psi_{R} D_{+} \Psi_{R}+D_{+} \Psi_{R} \Psi_{R}\right)
$$

These operator products are again singular and require point splitting. We find,

$$
P^{+}=\frac{\pi}{L} \sum_{n} n\left(a_{n}^{\dagger} a_{n}+b_{n}^{\dagger} b_{n}+d_{n}^{\dagger} d_{n}\right)-\frac{\pi}{L} Z_{R} Q_{R}+\frac{\pi}{2 L} Z_{R}^{2}
$$

One can explicitly show that this expression is $T_{1}$ and $R$ invariant. We will modifiy this express further below went we discuss the equation of motion and the conditions that define the physical subspace.

It is interesting to note that for the Noether form there would appear to be a very substantial contribution from the $x^{-}=0$ surface. The application of our rule for that term is somewhat unclear here because the term contains a derivative perpendicular to the $x^{+}=0$ surface. Using the equation of motion for $\Psi_{L}$ we find that the Noether form for $P^{+}$is

$$
P_{N}^{+}=\frac{\pi}{L} \sum_{n} n\left(a_{n}^{\dagger} a_{n}+b_{n}^{\dagger} b_{n}+d_{n}^{\dagger} d_{n}\right)+\frac{\pi}{L} \int_{-L}^{L} d x^{+} Z_{R}\left(x^{+}\right) \tilde{J}_{3}^{L}\left(x^{+}\right) .
$$

This form is of course not $T$ invariant. Furthermore $Z_{R}\left(x^{+}\right)$is initialized on $x^{+}=0$ and not on $x^{-}=0$ where the left handed contribution to $P^{+}$is calculated. Formally one can envisage solving for $Z_{R}\left(x^{+}\right)$in terms of the operator initialized on $x^{+}=0$ and then using it in the above expression. This of course raises the speculative nature of this form of $P^{+}$to an even higher level, and we only mention this form of $P^{+}$because it generates an interesting result in a later section.

\section{VACUUM STATES OF THE THEORY}

The Fock state containing no particles will be called $\left|V_{0}\right\rangle$. It is one of a set of states that are related to one another by $T_{1}$ transformations, and which will be denoted $\left|V_{M}\right\rangle$, where $M$ is any integer. These are defined by

$$
\left|V_{M}\right\rangle \equiv\left(T_{1}\right)^{M}\left|V_{0}\right\rangle
$$

where $\left(T_{1}\right)^{-1}=T_{-1}$. It is straightforward to determine the particle content of the $\left|V_{M}\right\rangle$. Consider, for example, the $T_{1}^{R}$ transform of

$$
b_{1 / 2}^{\dagger} b_{1 / 2}\left|V_{0}\right\rangle=0
$$


which is

$$
T_{1}^{R} b_{1 / 2}^{\dagger} T_{1}^{R-1} T_{1}^{R} b_{1 / 2} T_{1}^{R-1} T_{1}^{R}\left|V_{0}\right\rangle=0
$$

Using Eqn. (4.14) we have

$$
d_{1 / 2} d_{1 / 2}^{\dagger}\left|V_{1}\right\rangle=0
$$

which implies

$$
d_{1 / 2}^{\dagger} d_{1 / 2}\left|V_{1}\right\rangle=\left|V_{1}\right\rangle
$$

and therefore $\left|V_{1}\right\rangle$ will have one $d_{1 / 2}$ background particle. Similarly one could apply $T_{1}^{L}$ to

$$
\delta_{1 / 2}^{\dagger} \delta_{1 / 2}\left|V_{0}\right\rangle=0
$$

and one would conclude that $\left|V_{1}\right\rangle$ also contains one $\beta_{1 / 2}$ particle. One can show that $\left|V_{1}\right\rangle$ has no other content. Thus one finds that

$$
\left|V_{1}\right\rangle=d_{1 / 2}^{\dagger} \beta_{1 / 2}^{\dagger}|0\rangle
$$

We will focus here on the Fock states $\left|V_{0}\right\rangle$ and $\left|V_{1}\right\rangle$ since we will find that the degenerate vacuum state will be constructed from them. The combination $T_{1} R$, which is a symmetry of this theory, interchanges the Fock states $\left|V_{0}\right\rangle$ and $\left|V_{1}\right\rangle$ up to a phase,

$$
\begin{aligned}
& T_{1} R\left|V_{0}\right\rangle=(\text { phase })\left|V_{1}\right\rangle \\
& T_{1} R\left|V_{1}\right\rangle=(\text { phase })\left|V_{0}\right\rangle .
\end{aligned}
$$

It also might be possible to construct Bloch wave type states that sums over all domains as discussed in ref. 25] that are $T_{1} R$ invariant.

All of our states, as we noted previously, will be constructed from a Schrödinger wave function $\zeta_{0}\left(Z_{R}\right)$ and a Fock state. This wave function is the ground state of the Schrödinger equation obtained by projecting out the empty Fock state sector of the Hamiltonian [29].

We find that under $T_{1} R$ symmetry

$$
T_{1} R \zeta_{0}\left(Z_{R}\right)=(\text { phase }) \zeta_{0}\left(-Z_{R}-1\right)
$$

We chose the arbitrary phases such that,

$$
T_{1} R \zeta_{0}\left(Z_{R}\right)\left|V_{0}\right\rangle=e^{i \theta} \zeta_{0}\left(-Z_{R}-1\right)\left|V_{1}\right\rangle
$$

Then we can now construct the " $\Theta$ vacuum " for this theory that is invariant under the $T_{1} R$ symmetry,

$$
|\Omega\rangle=\frac{1}{\sqrt{2}}\left[\zeta_{0}\left(Z_{R}\right)\left|V_{0}\right\rangle+e^{i \theta} \zeta_{0}\left(-Z_{R}-1\right)\left|V_{1}\right\rangle\right]
$$

The state we have constructed in Eqn. 6.11) is an eigenstate: 


$$
T_{1} R|\Omega\rangle=|\Omega\rangle
$$

It is typically necessary to build the theory on such a vacuum state in order to satisfy the requirements of cluster decomposition as well.

It is straight forward to show that $|\Omega\rangle$ satisfies all the physical subspace conitions. At $x^{+}=0$ one can to show that

$$
\left\langle\Omega\left|Q_{R}-Z_{R}\right| \Omega\right\rangle=0
$$

independent of the precise form of the wave function $\zeta_{0}\left(Z_{R}\right)$ - the result only depends on the fact that the wavefunctions multiplying $\left|V_{0}\right\rangle$ and $\left|V_{1}\right\rangle$ are related in the way shown in (6.11). In addition, we must show that the matrix elements of all $x^{+}$derivatives of $Q_{R}-Z_{R}$ at $x^{+}=0$ (i.e., commutators of $Q_{R}-Z_{R}$ with $P^{-}$) vanish. This follows trivially, however, since $|\Omega\rangle$ is an eigenstate of $P^{-}$. Thus the condition (6.13) is satisfied for the vacuum state (6.11).

The question of whether there are excited states that satisfy (6.13) is more difficult. The energy eigenvalues of the system are proportional to $2 L$, as one would expect, since they correspond to fluctuation of the flux around the entire spatial volume. We will make a large $L$ approximation and only retain the ground state wave function $\zeta_{0}\left(Z_{R}\right)$

Then the physical states are constructed by applying right-handed, color singlet Fock operators to the ground state [26]. With this picture of physical states we can now consider the remaining anomaly condition Eq.(4.22). This condition on physical states (PS) is

$$
0=\left\langle P S\left|Q_{R}-Z_{R}\left(x^{+}\right)\right| P S\right\rangle \text {. }
$$

To satisfy Eq.(6.14) it is sufficient that the anomaly condition Eq.( 4.22) vanish between vacuum states, which we have already shown.

For this condition to hold at all times it is necessary that the matrix elements between physical states of all time derivatives of this equation also vanish. It is again sufficient that the first time derivative of $Z_{R}$ vanish between vacuum states,

$$
0=\left\langle\Omega\left|\Pi_{R}\right| \Omega\right\rangle
$$

This condition is again satisfied independent of the form of the wave function $\zeta_{0}\left(Z_{R}\right)$. The next condition one gets by taking the commutator of $\Pi_{R}$ with $P^{-}$. Since there are terms in $P^{-}$that depend on $Z_{R}$ this will produce the following operator.

$$
\sum_{N} \frac{\left\{C_{N}^{+}, C_{-N}^{-}\right\}}{\left(Z_{R}+N\right)^{3}} .
$$

The matrix elements of this operator between physical states are not zero and therefore the condition Eq. 6.14) in not entirely stable. This is to be expected because of the large- $L$ approximation that we made. If our physical subspace formed a complete set of energy eigenstates then the vanishing of all matrix elements of $\Pi_{R}$ would assure the vanishing of all matrix element of the commutators of $P^{-}$with $\Pi_{R}$. However, since $P^{-}$is $Z_{R^{-}}$dependent it will generate transitions from the ground state $\zeta_{0}\left(Z_{R}\right)$ to the excited flux states with 
energies proportional to $2 L$ which we have excluded. Neglecting these states necessarily destroys the stability. Since all the states that we neglected are high energy states which decouple in the large $L$ limit, the the instability presumably disappears in the large $L$ limit. It is interesting to also remark that in the absence of matter the operator $\Pi_{R}$ is constant 20] and this problem does not arise.

\section{EQUATIONS OF MOTION}

We have obtained expressions for $P^{+}$and $P^{-}$in the previous section but we can not be sure that they are correct for this theory until we show that they generate the Dirac equation and Ampere's law for this theory.

In matrix form, the Dirac equations of motion for the theory are

$$
\begin{aligned}
& D_{+} \Psi_{R}=\partial_{+} \Psi_{R}+i g\left[A, \Psi_{R}\right]=0 \\
& D_{-} \Psi_{L}=\partial_{-} \Psi_{L}+i g\left[V, \Psi_{L}\right]=0
\end{aligned}
$$

It is straightforward to resolve these into color components, using the helicity basis described above, and implementing the gauge we have chosen. Eqns. (7.1) and (7.2) become

$$
\begin{aligned}
& \partial_{-} \psi_{L}=0 \\
& \partial_{-} \phi_{L}=-i g v \phi_{L} \\
& \partial_{+} \psi_{R}=i g\left[A_{-} \phi_{R}-A_{+} \phi_{R}^{\dagger}\right] \\
& \partial_{+} \phi_{R}=i g\left[A_{+} \psi_{R}-A_{3} \phi_{R}\right] .
\end{aligned}
$$

The first two equations evolve $\Psi_{L}$ in $x^{-}$, off of its initial-value surface, while the second pair determine the $x^{+}$-evolution of $\Psi_{R}$.

Ampere's law,

$$
D_{+} D_{-} A=g J^{-},
$$

requires particularly careful consideration in this theory since it explicitly connects left- and right-handed quantities. It decomposes into

$$
\begin{gathered}
\partial_{+} \partial_{-} A_{3}-\partial_{+}^{2} v+i g\left[A_{+}\left(\partial_{-}-i g v\right) A_{-}-A_{-}\left(\partial_{-}+i g v\right) A_{+}\right]=g J_{3}^{-} \\
\partial_{+}\left[\left(\partial_{-} \pm i g v\right) A_{ \pm}\right] \pm i g A_{ \pm} \partial_{+} v \pm i g\left[A_{3}\left(\partial_{-} \pm i g v\right) A_{ \pm}-A_{ \pm} \partial_{-} A_{3}\right]=g J_{ \pm}^{-}
\end{gathered}
$$

Checking these is a straightforward exercise in commuting fields with $P^{ \pm}$and comparing the results with the corresponding equations of motion. We simply summarize the results here. 
It turns out that the equation of motion for $\psi_{R}$ is satisfied if

$$
Z_{L}=-Q_{R}
$$

which we shall take to be a strong (operator) equality. This determines the zero mode of $A_{3}$, which we were not able to fix using Gauss' law. In addition, the color 3 component of Ampere's law, Eqn. (7.8), leads to

$$
Z_{L}=Q_{L}
$$

which, when combined with Eqn. (7.10), gives

$$
Q_{R}+Q_{L}=0
$$

We shall impose this as an eigenvalue condition on states. Ampere's law also leads to the conditions

$$
D_{N}^{3}=D_{N}^{ \pm}=0
$$

which must be realized in matrix elements between states. We shall therefore require physical states to satisfy

$$
D_{N}^{3}|\Phi\rangle=D_{N}^{ \pm}|\Phi\rangle=0 \quad(N>0)
$$

Finally, Ampere's law requires the condition (4.22) which we discussed in the previous section.

We can now modify $P^{ \pm}$so that the kinematic Heisenberg equations are satisfied by replacing troublesome terms with operators to which they are weakly equivalent. In Eqn. (5.11) we substitute the operator relation $(\overline{7.10})$ and replace $Q_{L} \rightarrow-Q_{R}$, as suggested by Eqn. (7.12). The result is

$$
P_{l h}^{-}=\frac{\pi}{L} \sum_{n} n\left(\alpha_{n}^{\dagger} \alpha_{n}+\beta_{n}^{\dagger} \beta_{n}+\delta_{n}^{\dagger} \delta_{n}\right)-\frac{\pi}{2 L} Q_{R}^{2},
$$

which is consistent with the kinematical Heisenberg relation for $\phi_{L}$. Similarly, in Eqn. (5.14) we can replace $Z_{R} \rightarrow-Q_{L}$ and $Q_{R} \rightarrow-Q_{L}$ to obtain

$$
P^{+}=\frac{\pi}{L} \sum_{n} n\left(a_{n}^{\dagger} a_{n}+b_{n}^{\dagger} b_{n}+d_{n}^{\dagger} d_{n}\right)-\frac{\pi}{2 L} Q_{L}^{2} .
$$

This expression is consistent with all relevant Heisenberg equations. Note that the replacement $Z_{R} \rightarrow-Q_{L}$, which might have been expected to be inconsistent with the Dirac equation for $\phi_{L}$, is in fact consistent in the subspace defined by (4.22). Thus this modification of $P^{+}$ does not lead to any new conditions on states.

The final result is that with $P^{+}$given by Eqn. (7.16) and $P^{-}$given by the sum of $(7.15)$ and $(\underline{5.12})$, all Heisenberg equations are correctly obtained in the subspace defined by (7.12), (7.14), and (4.22).

Let us now discuss the axial anomaly in this theory, 


$$
\partial_{\mu} J_{5}^{\mu, 3}=\frac{g}{2 \pi} \epsilon^{\mu \nu} F_{\mu \nu}^{3},
$$

where $J_{5}^{\mu}=\frac{-1}{\sqrt{2}}\left[\Psi, \gamma^{\mu} \gamma^{5} \Psi\right]$ and is related to the vector current through $J_{5}^{\mu}=\left(J^{R},-J^{L}\right)$. It can be shown that covariant derivatives reduce to partial derivatives for $J^{\mu, 3}$ so the conservation equations below take the Abelian from for the matter currents. We can calculate $\partial_{+} \tilde{J}_{3}^{R}$ from

$$
i\left[P^{-}, \tilde{J}_{3}^{R}\right]=\partial_{+} \tilde{J}_{3}^{R}(x)
$$

Using

$$
\left[\tilde{J}_{3}^{R}\left(0, x^{-}\right), \tilde{J}_{3}^{R}\left(0, y^{-}\right)\right]=\frac{i}{2 \pi} \delta^{\prime}\left(x^{-}-y^{-}\right)
$$

and we find

$$
\partial_{+} \tilde{J}_{3}^{R}=\frac{g}{2 \pi} \partial_{-} A_{3} .
$$

Combining this with the gauge correction for $J_{3}^{R}$ we find;

$$
\partial_{+} J_{3}^{R}=\frac{g}{2 \pi}\left(\partial_{-} A_{3}-\partial_{+} v\right) .
$$

Now $\tilde{J}_{3}^{L}$ is explicitly independent of $x^{-}$by the equation of motion for $\Psi_{L}$. However this is deceptive and we see that if we calculate $\partial_{-} \tilde{J}_{3}^{L}$ from $i\left[P^{+}, \tilde{J}_{3}^{L}\right]$ and use Eq.(5.15) and

$$
\left[\tilde{J}_{3}^{L}\left(x^{+}, 0\right), \tilde{J}_{3}^{L}\left(y^{+}, 0\right)\right]=\frac{i}{2 \pi} \delta^{\prime}\left(x^{+}-y^{+}\right),
$$

then we find,

$$
\partial_{-} \tilde{J}_{3}^{L}=\frac{g}{2 \pi} \partial_{+} v \approx 0,
$$

If we had used the correct forms of $P^{+}$,Eq.(7.16), we would not find this contribution. On the other hand $\partial_{+} v$ is $\Pi_{R}$ and we have already discussed the fact that all matrix elements of it between physical states vanish, and this is the reason for the apparent asymmetry between Eq.(7.23) and Eq.(7.20). If we retain this term and combine it with the gauge correction to $J_{3}^{L}$ the complete result for $\partial_{-} J_{3}^{L}$ is;

$$
\partial_{-} J_{3}^{L}=-\frac{g}{2 \pi}\left(\partial_{-} A_{3}-\partial_{+} v\right) .
$$

Therefore

$$
\partial_{\mu} J_{3}^{\mu}=\partial_{+} J_{3}^{R}+\partial_{-} J_{3}^{L}=0
$$

and

$$
\partial_{\mu} J_{3}^{5, \mu}=\partial_{+} J_{3}^{R}-\partial_{-} J_{3}^{L}=\frac{g}{\pi}\left(\partial_{-} A_{3}-\partial_{+} v\right)=\frac{g}{2 \pi} \epsilon^{\mu \nu} F_{\mu \nu}^{3},
$$

as expected. 


\section{THE CONDENSATE}

It is generally accepted that $Q C D$ in $1+1$ dimensions coupled to adjoint fermions develops a condensate $\Sigma \equiv\langle\Omega|\bar{\Psi} \Psi| \Omega\rangle$. Thus far $\Sigma$ has been calculated in the large- $N_{c}$ limit [27] and in the small-volume limit for $S U(2)$ in Ref. [15]. Previously we calculated a condensate in the chiral theory with only right-handed fermions [28]. In that calculation it was the field itself that had a condensate and the result was fundamentally different from what we are considering here.

The two vacuum states $\zeta_{0}\left(Z_{R}\right)\left|V_{0}\right\rangle$ and $\zeta_{0}\left(-Z_{R}-1\right)\left|V_{1}\right\rangle$ are exact ground states. We have a spectral flow associated with the right- and left-handed operators, and thus the two physical spaces in the fundamental domain differ by one right-handed and one left-handed fermion. This effectively block diagonalizes $P^{-}$into two sectors, one of which is built on a state with no background particles and one of which is built on a state with background particles. The two sectors communicate through the condensate. Physical states must respect the $T_{1} R$ symmetry and must therefore be built on the " $\theta$ vacuum" $|\Omega\rangle$, which is a linear combination of these two vacuum states.

To calculate the condensate $\Sigma$ let us consider $\operatorname{Tr}(\bar{\Psi}(0) \Psi(0))$ and retain only the terms which can contribute to the vacuum expectation value:

$$
\operatorname{Tr}(\bar{\Psi}(0) \Psi(0))=-\frac{i}{2 L \sqrt{2}}\left[d_{1 / 2}^{\dagger} \beta_{1 / 2}^{\dagger}+d_{1 / 2} \beta_{1 / 2}\right]+\ldots
$$

We chose to evaluate $\Sigma$ at the point $(0,0)$ because it is the point where the two initial value surfaces intersect. Because of the approximation we have made we are unable to preform the calculation away from this point. Taking the expectation value in $|\Omega\rangle$ we find that only the cross terms contribute. We thus obtain for the vacuum expectation value of $\bar{\Psi} \Psi$ from Eqn. (6.11):

$$
\Sigma=-\frac{\sin \theta}{2 L \sqrt{2}} \int_{-1}^{0} \zeta_{0}\left(Z_{R}\right) \zeta_{0}\left(-Z_{R}-1\right) d Z_{R}
$$

Eqn. 6.11) is an exact expression for the vacuum, Eqn. (8.2) is an exact expression for $\Sigma$. We see that this expression behaves like $1 / L$. This is a common result for discrete light cone calculations and is found even in the Schwinger model where the exact result is known not to have this behavior. We also note that the only other calculation [15] of this quantity, done in different gauge, in a small volume approximation and discretized and quantized at equal time, finds this $1 / L$ behavior.

In 29] the explicit form of the Hamiltonian for ground state wave function is given and solved numerically. Using this form of numerical solution the value for the condensate is calculated.

We have not addressed directly the question of whether the condensate affects the spectrum of this theory but clearly it raises a number of interesting questions. It is interesting to consider this in light of work of Kutasov and Schwimmer [11. They claim that there

are classes of theories that have the same massive spectrum. The simplified version of the their argument goes as follows: In light-front quantization of $Q C D_{1+1}$ coupled to massless fermions, the left- and right-moving fermions decouple and the right-movers alone generate 
the massive spectrum. The role of left-moving particles is simply to cancel the anomaly and balance the total charge of states. Thus one could in general choose any left-handed particles that accomplish this. For example, in the theory considered here (one adjoint fermion) we could just as well take for the left-handed fields two flavors of fundamental fermions. Then one argues, based on Lorentz and gauge invariance, that one could study this new theory in the gauge $A^{-}=0$ and using $x^{-}=0$ as the principal quantization surface. In this case the roles of the left- and right-moving particles are reversed, and the spectrum is now generated by the left-handed particles. The massive spectra (as well as scattering amplitudes for massive states) in these models should therefore be the same. For example, $Q C D_{1+1}$ coupled to two flavors of fundamental fermions should have the same massive spectrum as a single flavor of adjoint fermions. However the theory with fundamental fermions will not have a condensate.

One of the conditions for this universality is the decoupling of the left- and right-handed fields. This follows because in the basis built on $\left|V_{0}\right\rangle$ and $\left|V_{-1}\right\rangle, P^{-}$is block diagonal. Furthermore, using the $T_{1} R$ symmetry one can show that the blocks are identical. Therefore the condensate does not affect the massive spectrum of the theory.

Kutusov and Schwimmer also point out that left- and right-handed fermions do not decouple if one regulates the theory using an equal-time spatial circle (i.e., anti-periodic boundary conditions on $x^{1}$ ) as used in [15]. In simple terms the argument is that when one rolls the coordinate $x^{1}$ the lines $x^{+}=$constant along which the right-handed fermions propagate and the lines $x^{-}=$constant along which the left-handed fermion propagate will intersect an infinite number of times. By contrast, in flat space and with a circle in $x^{-}$these line only intersect once. Thus this method of regularization is more natural.

The claims of Kutusov and Schwimmer have never been numerically checked and we hope that this calculation is the first step in that direction. Checking these relations would present a particularly interesting challenge for lattice gauge theory since these are relations between theories with even and odd numbers of fermions. Among other interesting results we would expect to see is the screening of "fractional" charges, e.g., charges in the fundamental representation of $S U(N)$ when the dynamical fermions are in the adjoint representation [16]. It has also been shown that when the dynamical fermions are given a mass the screening is replaced by true confinement, with a string tension given by

$$
\sigma=2 m \Sigma
$$

This relation also indirectly shows the deconfinement at zero mass [16].

\section{CONCLUSIONS}

We have shown that in $Q C D$ coupled to adjoint fermions in two dimensions the lightfront vacuum is two-fold degenerate as one would expect on general grounds. The source of this degeneracy is quite simple. Because of the existence of Gribov copies, the one gauge degree of freedom, the zero mode of $A^{+}$, must be restricted to a FMD. The domain of this variable, which after normalization we call $Z_{R}$, is bounded by the integers. Furthermore there is a $T_{1} R$ symmetry which for $Z_{R}$ is simply a reflection about the midpoint of the FMD. The $T_{1} R$ symmetry operator acting on the fermionic Fock vacuum generates a second 
degenerate state. Since $T_{1}$ generates a spectral flow for the fermions this second vacuum contains one left-handed and one right-handed fermion each with momentum $\pi / 2 L$, but the state can be shown to still have $P^{+}=P^{-}=0$. We form the analog of a $\theta$ vacuum from these degenerate vacuum states which respects all of the symmetries of the theory.

We found that the zero mode of $J_{3}^{R}$ includes a gauge correction $Z_{R}\left(x^{+}\right)$, and since $Z_{R}\left(x^{+}\right)$ is dynamical it is impossible to satisfy the zero mode of Gauss' law for the color three component. Furthermore this problem reappears in the \pm color components of Ampere's law. We show that this condition on the zero mode of $J_{R}^{3}$ can hold weakly as a condition on matrix elements between physical states in the large $L$ limit.

We find that $\operatorname{Tr}(\bar{\Psi} \Psi)$ has a vacuum expectation value with respect to this $\theta$ vacuum and we find an exact expression for this condensate $\Sigma$. It is unlikely that the condensate we find here is equivalent to that found in [15], because the infrared regulator used there appears to couple the left and right-handed fermions.

Since $P^{-}$is block diagonal in our degenerate vacuum states the condensate does not affect the massive spectrum of the theory and the theory has no massless bound state. However this

condensate is proportional to the string tension characterizing the force between fractional charges when the adjoint fermions are given a small mass.

\section{ACKNOWLEDGMENTS}

This work was supported in part by a grant from the US Department of Energy. S.S.P would like to acknowledge the hospitality of the Aspen Center for Physics where part of this work was completed, David Kutasov for many helpful and instructive conversations and Syd Meshkov for a careful reading of the manuscript. 


\section{REFERENCES}

[1] P. A. M. Dirac, Rev. Mod. Phys. 21, 392 (1949).

[2] H.-C. Pauli and S. J. Brodsky, Phys. Rev. D 32, 1993, 2001 (1985).

[3] T. Maskawa, and K. Yamawaki, Prog. Theor. Phys. 56, 270 (1976).

[4] T. Heinzl, S. Krusche, S. Simbürger and E. Werner, Z.Phys. C 56, 415 (1992).

[5] D. G. Robertson, Phys. Rev. D 47, 2549 (1993).

[6] C. M. Bender, S. Pinsky and B. van de Sande, Phys. Rev. D48, 816 (1993).

[7] S. S. Pinsky and B. van de Sande, Phys. Rev. D 49, 2001 (1994).

[8] J. Hiller, S.S. Pinsky and B. van de Sande, Phys.Rev. D51, 726 (1995).

[9] E. Witten, Nuovo Cim. A 51, 325 (1979).

[10] A. Smilga, Phys. Rev. D 49, 6836 (1994).

[11] D. Kutasov and A. Schwimmer, Nucl. Phys. B 442, 447 (1995).

[12] N. Seiberg and E. Witten, Nucl. Phys. B 426, 19 (1994).

[13] G. McCartor, Z. Phys. C 41, 271 (1988).

[14] G. McCartor, D. G. Robertson and S. S. Pinsky, "Vacuum Structure of TwoDimensional Gauge Theories on the Light Front," hep-th/9609???.

[15] F. Lenz, M. Shifman and M. Thies, Phys. Rev. D 51, 7060 (1995).

[16] D. J. Gross, I. R. Klebanov, A. V. Matytsin and A. Smilga, Nucl. Phys. B 461, 109 (1996).

[17] S. Pinsky, "Topology and Confinement in Light-Front QCD," in Theory of Hadrons and Light-Front QCD, St. D. Glazek, ed. (World Scientific, 1995).

[18] P. Goddard and D. Olive, Int. J. Mod. Phys. A 2, 303 (1986).

[19] V. A. Franke, Yu. A. Novozhilov and E. V. Prokhvatilov, Lett. Math. Phys. 5, 239, 437 (1981); and in Dynamical Systems and Microphysics (Academic Press, 1982).

[20] A. C. Kalloniatis, H.-C. Pauli and S. S. Pinsky, Phys. Rev. D 50, 6633 (1994).

[21] S. S. Pinsky and A. Kalloniatis, Phys. Lett. B 365, 225 (1995).

[22] V. N. Gribov, Nucl. Phys. B 139, 1 (1978).

[23] F. Lenz, M. Thies, S. Levit, and K. Yazaki, Ann. Phys. (N.Y.) 208, 1 (1991).

[24] G. McCartor, hep-th/9410197, in Theory of Hadrons and Light-Front QCD, St. D. Glazek, ed. (World Scientific, 1995).

[25] E. Langmann and G. Semenoff $Q C D_{1+1}$ With Massless Quarks and Gauge Covariant Sugawara Construction"

[26] K. Demeterfi, I. R. Klebanov, and G. Bhanot, Nucl. Phys. B 418, 15 (1994); G. Bhanot, K. Demeterfi, and I. R. Klebanov, Phys. Rev. D 48, 4980 (1993);

[27] I. I. Kogan and A. R. Zhitnitsky, OUTP-95-25 P, hep-ph/9509322.

[28] S. S. Pinsky and D. G. Robertson, Phys. Lett. B 379, 169 (1996).

[29] S. S. Pinsky and R. Mohr, to appear in Int. J. Mod. Phys. 